This item was submitted to Loughborough's Research Repository by the author.

Items in Figshare are protected by copyright, with all rights reserved, unless otherwise indicated.

\title{
Phase behavior of a fluid with competing attractive and repulsive interactions
}

PLEASE CITE THE PUBLISHED VERSION

http://dx.doi.org/10.1103/PhysRevE.76.031501

\section{PUBLISHER}

(C) The American Physical Society

\section{VERSION}

VoR (Version of Record)

\section{PUBLISHER STATEMENT}

This work is made available according to the conditions of the Creative Commons Attribution-NonCommercialNoDerivatives 4.0 International (CC BY-NC-ND 4.0) licence. Full details of this licence are available at: https://creativecommons.org/licenses/by-nc-nd/4.0/

\section{LICENCE}

CC BY-NC-ND 4.0

\section{REPOSITORY RECORD}

Archer, Andrew J., and Nigel B. Wilding. 2019. "Phase Behavior of a Fluid with Competing Attractive and Repulsive Interactions". figshare. https://hdl.handle.net/2134/16037. 


\title{
Phase behavior of a fluid with competing attractive and repulsive interactions
}

\author{
Andrew J. Archer ${ }^{1,2}$ and Nigel B. Wilding ${ }^{2}$ \\ ${ }^{1}$ Department of Mathematical Sciences, Loughborough University, Loughborough LE11 3TU, United Kingdom \\ ${ }^{2}$ Department of Physics, University of Bath, Bath BA2 7AY, United Kingdom
}

(Received 5 June 2007; published 4 September 2007)

\begin{abstract}
Fluids in which the interparticle potential has a hard core, is attractive at moderate separations, and repulsive at large separations are known to exhibit unusal phase behavior, including stable inhomogeneous phases. Here we report a joint simulation and theoretical study of such a fluid, focusing on the relationship between the liquid-vapor transition line and any new phases. The phase diagram is studied as a function of the amplitude of the attraction for a certain fixed amplitude of the long ranged repulsion. We find that the effect of the repulsion is to substitute the liquid-vapor critical point and a portion of the associated liquid-vapor transition line, by two first-order transitions. One of these transitions separates the vapor from a fluid of spherical liquidlike clusters; the other separates the liquid from a fluid of spherical voids. At low temperature, the two transition lines intersect one another and a vapor-liquid transition line at a triple point. While most integral equation theories are unable to describe the new phase transitions, the Percus-Yevick approximation does succeed in capturing the vapor-cluster transition, as well as aspects of the structure of the cluster fluid, in reasonable agreement with the simulation results.
\end{abstract}

DOI: 10.1103/PhysRevE.76.031501

PACS number(s): 61.20.-p, 05.70.Fh, 61.46.Bc

\section{INTRODUCTION}

Until quite recently, it was commonly supposed that the gamut of equilibrium fluid phase behavior exhibited by a single component system of particles interacting via an isotropic pair potential, does not extend beyond a liquid phase and a vapor phase. While this certainly appears to be true for prototype models such as the Lennard-Jones fluid, it is now recognized that considerably richer phase behavior can occur [1]. For instance, systems whose particles have a repulsive core that is sufficiently soft, may exhibit one or more stable liquid-liquid phase transitions over and above the liquidvapor transition [2-4]. Furthermore, and notwithstanding their isotropy, such soft potentials can give rise to inhomogeneous fluid phases composed of clusters, or patterned morphologies such as stripes and lamellae [5-9].

Another class of isotropic pair potential exhibiting rich phase behavior are those that are attractive for short distances (beyond a hard core diameter) but repulsive at large distances, i.e., they are short-range attractive and long-range repulsive (SALR). Effective potentials of the SALR form can be found in colloid-polymer mixtures [10-13]: The long ranged repulsion arises from the weakly screened charge carried by the colloids, while the attraction at short distances stems from the depletion forces associated with the nonadsorbing polymers [14]. The competition between attraction and repulsion on distinct length scales is responsible for behavior such as the appearance of equilibrium cluster phases and nonequilibrium gel states $[11,12]$. SALR potentials are further appropriate for the description of protein solutions $[10,15]$, star-polymer systems [16], the effective interactions between solute particles in a subcritical liquid solvent [17], and colloidal monolayers [18,19]. In the latter (twodimensional) systems, the short-range attraction arises from the van der Waals and capillary forces, while the longer range repulsion is thought to be due to dipole-dipole interactions $[18,19]$. Such two-dimensional systems have also been observed to form cluster and stripe morphologies [18-21].

Beyond their relevance to the real systems listed above, models whose particles interact via a SALR potential are of considerable interest from the fundamental perspective of statistical mechanics. Specifically, they elicit basic questions such as the following: What is the scope and character of their phase behavior, and how can this be described theoretically? Below we briefly review the progress to date in addressing these issues.

A number of theoretical and simulation studies have considered aspects of the phase behavior of a variety of fluids interacting via SALR potentials [22-40]. In pioneering early work [22-24], a mean-field (Landau) theory approach was developed for systems with competing interactions. This predicted that when the amplitude of the long ranged repulsion is sufficiently large (relative to that of the attraction), modulated phases appear in the region of the phase diagram where, if one were to allow only for the occurrence of homogeneous phases, the theory would predict the liquid-vapor critical point to be located. More recent field-theoretic studies [25-27] have arrived at similar conclusions.

Adopting a phenomenological approach, Groenewold and Kegel $[29,30]$ developed a model to explain how competition between short ranged attraction and a longer ranged repulsion in colloidal systems could promote cluster formation. They concluded that depending on the relative strengths and ranges of the competing attractive and repulsive contributions to the pair potential, large clusters (up to several thousands of particles) would be stable. Signatures of such clustering were observed by Sear and Gelbart within a meanfield [random phase approximation (RPA)] liquid state theory [28] study of a model in which the attractive and repulsive contributions to the potential are both rather long ranged (justifying the mean-field approximation). They showed that the propensity to clustering is manifest by a peak in the static structure factor, $S(k)$, at a small but nonzero wave vector $k_{c}$. In fact, the mean-field theory predicts a line in the phase 
diagram at which $S(k)$ diverges at $k=k_{c} \neq 0$, and it was inferred that this line indicates microphase separation to a modulated phase(s). Such a line has previously been dubbed the " $\lambda$ line" [32] in terminology borrowed from other contexts [41-43].

Within the framework of integral equation theory, Chen and co-workers [33-35] solved the Ornstein-Zernike (OZ) equation for the structure of a fluid of particles interacting via a SALR potential in which the attractive and repulsive contributions are both assigned the Yukawa form (a "doubleYukawa" potential) [44-46]. Two closures were examined: the mean spherical approximation (MSA) and the hypernetted-chain (HNC) approximation. Focusing on the portion of the phase diagram where the peak in $S(k)$ at $k$ $=k_{c}$ is developing, the authors found that both theories provide a good description of the fluid structure, as gauged by comparison with Monte Carlo (MC) simulations. As will be demonstrated in the present work, however, neither the MSA nor the $\mathrm{HNC}$ is reliable for describing the fluid structure in the vicinity of transitions to inhomogeneous phases.

A generally more accurate integral equation theory, the self-consistent Ornstein-Zernike approximation (SCOZA), has been applied in conjunction with hierarchical reference theory (HRT) calculations, by Pini et al. $[31,47]$ to a doubleYukawa SALR potential. The authors investigated the influence on the structure and phase behavior of the fluid as the amplitude of the repulsive contribution was increased from zero. Doing so was found to depress the vapor-liquid critical temperature and led to the appearance of an anomalously large region around the liquid-vapor critical point in which the fluid compressibility is very high [31,47]. Unfortunately, Pini et al. were unable to obtain solutions either from the SCOZA or the HRT in the regime where inhomogeneous phases might be expected to occur $[31,32,47]$. The SCOZA results were subsequently compared with a RPA density functional theory (DFT) by Archer et al. [32]. In common with earlier mean-field approaches [28], the RPA DFT predicts a $\lambda$ line to occur in the phase diagram when the amplitude of the repulsive part of the potential exceeds a threshold value [32]. In parts of the phase diagram away from the $\lambda$ line, reasonable agreement with SCOZA was found for the fluid structure and thermodynamics.

Sciortino and co-workers [36-38] have employed a variety of theoretical and simulation techniques to investigate the properties of SALR systems. They focused attention on potentials having a particularly short ranged attractive part (i.e., a small fraction of the particle diameter), chosen to mimic the effective pair potential in certain colloidal systems. They found - as has been shown experimentally [12] — that the system exhibits both a fluid cluster phase and a gel phase comprising interconnected chains composed of face-sharing tetrahedral clusters. Indeed, as has been recently emphasized $[27,40]$, it is a general feature of models having particularly short ranged attraction, that the microphase separation can occur in a regime where the system dynamics are very slow and a transition to a nonergodic state is possible. Another SALR potential having a similarly short ranged attraction was studied using simulation by de Candia et al. who observed columnar and lamellar phases [39]. It has even been suggested that for such very short ranged attractive poten- tials, the presence of a long ranged repulsion might not be a prerequisite for cluster formation [48].

Notwithstanding the extensive body of impressive results on a variety of model SALR systems, displaying an intriguing wealth of inhomogeneous phases, central questions remain unanswered. Specifically, the detailed relationship between the liquid-vapor transition and the inhomogeneous phases still seems obscure (recall that mean-field theories predict a $\lambda$ line enclosing a region of the phase diagram in which a naive application of the theory would predict the liquid-vapor critical point to be [32]). Furthermore, if the vapor-liquid critical point is lost when long ranged repulsion is introduced, what happens to the remainder of the liquidvapor transition line? In the present work, we attempt to answer these questions by deploying simulation and theory to investigate the structure and phase behavior of a model system whose particles interact via a double-Yukawa SALR potential.

Our principal findings are as follows. Our Monte Carlo simulations show that for a certain (moderately large) strength of the repulsive contribution to the pair potential, the liquid-vapor critical point is absent. In its stead we find two lines of first-order phase transitions, each of which separates a homogeneous phase from an inhomogeneous (cluster) phase. One of these two transition lines is located at low particle number density, and separates the vapor from a fluid of spherical liquidlike clusters; it appears to terminate at a critical point at high temperatures. The other line-located at high density - separates a phase of spherical voids from the homogeneous liquid; it too appears to terminate at a critical point. At low temperature, the two transition lines intersect one another and a vapor-liquid transition line at a triple point.

We complement our simulation studies with an investigation of the utility of a number of standard liquid state theories for describing the phase behavior of our model. We first apply the DFT of Ref. [32] to trace the locus of the $\lambda$ line, noting that if one interprets this line as representing the transition to periodically modulated phases, then its topology is incompatible with the phase diagram emerging from the simulations. Turning our attention to integral equation theories, we find that the $\mathrm{HNC}$ has a no-solution region in the portion of the phase diagram where the transitions to inhomogeneous phases occur, although it does yield a portion of the vapor-liquid transition. Use of a simple modified HNC (MHNC) approximation similarly fails to provide a solution in the region of interest. We further find that (for separate reasons) the MSA is of little use in this region of the phase diagram. Interestingly, however, the Percus-Yevick (PY) approximation is able to describe the vapor-cluster phase transition as well as key aspects of the structure of the cluster fluid, in reasonable agreement with the MC results.

Our paper is organized as follows. We introduce our model SALR potential in Sec. II. The MC simulation methodology used to study the model, and our findings concerning the phase behavior and the character of the inhomogeneous phases are described in Sec. III. An investigation of the utility of mean-field DFT and integral equations for describing the phase behavior is detailed in Sec. IV. Finally, a discussion of the implications of our findings and the outlook for future work are the features in Sec. V. 


\section{MODEL}

The model that we have elected to study comprises an isotropic two-body interparticle potential of the hard-core plus double-Yukawa form,

$$
\beta v(r)= \begin{cases}\infty, & r \leq \sigma, \\ -\epsilon \sigma \exp \left[-z_{1}(r / \sigma-1)\right] / r & \\ +A \sigma \exp \left[-z_{2}(r / \sigma-1)\right] / r, & r>\sigma .\end{cases}
$$

Here $\beta=1 / k_{B} T$ is the inverse temperature, which we set equal to unity, while $\sigma$ is the particle diameter. The first Yukawa term represents an interparticle attraction whose strength is controlled by a parameter $\epsilon>0$, while the second term, with strength parameter $A>0$, represents a repulsion. We shall focus on the regime in which the range of the repulsion exceeds that of the attraction, i.e., $z_{1}>z_{2}$, leading to a repulsive tail to the potential.

The specific choice of $z_{1}$ and $z_{2}$ requires a balance to be struck between a number of competing desiderata. First, the attractive part should not be so short ranged that the equilibrium liquid-vapor phase behavior is wholly preempted by freezing $[47,49]$ or dynamic arrest (gelation) [40], phenomena which-while undoubtedly of interest in their own right - are not the specific concern of this work. Second, the range of the repulsive part must exceed that of the attractive contribution, and be sufficiently large to enable meaningful comparisons with mean-field theories, the accuracy of which increases with the potential range. Finally, from a purely practical standpoint, the range should not be so great that very large system sizes are necessary in order to obviate cutoff artifacts when the potential is truncated in the interests of computational efficiency. A satisfactory compromise in these respects was found by assigning $z_{1}=2, z_{2}=1$, and truncating the potential at $r=r_{c}=7.0 \sigma$. For reasons relating to the occurrence of large length-scale inhomogeneous phases, no mean-field correction was applied for the effects of the potential truncation, since such corrections assume the system is homogeneous.

As described in the Introduction, physically one can regard the potential of Eq. (1) as capturing the combined overall effect of a screened Coulombic repulsion between charged colloidal particles, and a shorter ranged attraction engendered by a depletion agent such as polymer chains. In this spirit, it is natural to regard $\epsilon$ as a measure of the polymer fugacity, $A$ as a measure of the colloidal charge, and treat both parameters independently. In the present work, however, we shall primarily be concerned with the phase behavior as a function of $\epsilon^{-1}$ at fixed $A$. For the latter, we have assigned the value $A=0.55$. This choice was motivated by a preliminary study of the model within DFT [32], which suggested that (given the choice $z_{1}=2, z_{2}=1$ ) this strength of repulsion is sufficient to engender a $\lambda$ line encompassing the critical region of the liquid-vapor line. One might therefore hope that qualitative alterations to the standard scenario of liquid-vapor phase behavior would ensue.

The form of the potential $\beta v(r)$ is shown in Fig. 1 for parameter values $\epsilon^{-1}=0.4, A=0.55, z_{1}=2, z_{2}=1$. Also shown is the form of $(r / \sigma)^{2} \beta v(r)$, which provides a useful indication of which cutoff values, $r_{c}$, are likely to result in signifi-

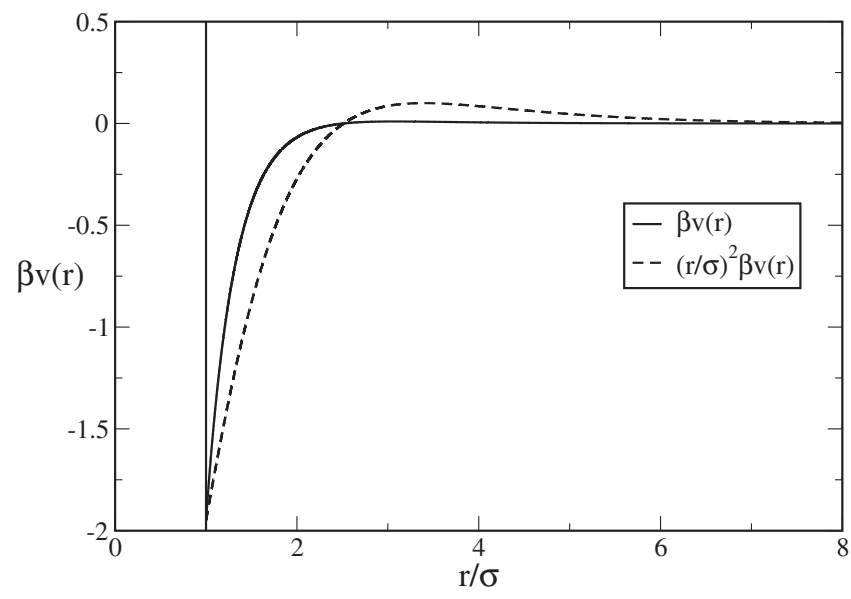

FIG. 1. The form of the potential $\beta v(r)$ [Eq. (1)] for parameters $\epsilon^{-1}=0.4, A=0.55, z_{1}=2, z_{2}=1$. Also shown for comparison is the form of $(r / \sigma)^{2} \beta v(r)$.

cant corrections to the internal energy compared to the full potential. The figure confirms that for our choice of the cutoff, $r_{c}=7.0 \sigma$, only small corrections to the limiting behavior are to be expected. It should be noted, however, that because this cutoff value is much larger than the values typically employed in the simulation of simple fluids such as the Lennard-Jones system, any simulation study is expected to entail a considerable computational investment.

\section{SIMULATION STUDIES}

\section{A. Techniques}

We have studied the phase behavior of the model of Sec. II using a grand canonical MC simulation algorithm [50]. Where possible, accurate location of points of first-order phase coexistence was facilitated by the use of the multicanonical preweighting technique [51], aided by multihistogram reweighting [52] according to the procedure described in Ref. [53]. Most of the results we shall present were obtained for a system of linear size $L=21 \sigma$, although some data was also collected for $L=28 \sigma$ in order to gauge the scale of finite-size effects. Periodic boundaries were employed throughout.

As shall be described below, techniques were implemented to determine the distribution of sizes of particle clusters in selected regions of the phase diagram. A cluster comprises a subset of particles that are interlinked via pathways of interparticle bonds. However, in contrast to lattice models, the definition of a bond in a system with continuous translational symmetry is somewhat ambiguous. We adopt a criterion which derives from that used for cluster identification in spin models. Specifically, we determine the interaction energy $v$ between each pair of particles and assign a bond with probability $p_{\text {bond }}=1-\exp (\beta v)$. Clusters of bonded particles are then identified using the efficient enumeration algorithm of Hoshen and Kopelman [54].

\section{B. Phase diagram}

Prior to commencing exploration of the phase diagram, an initial estimate was required for the range of values of the 

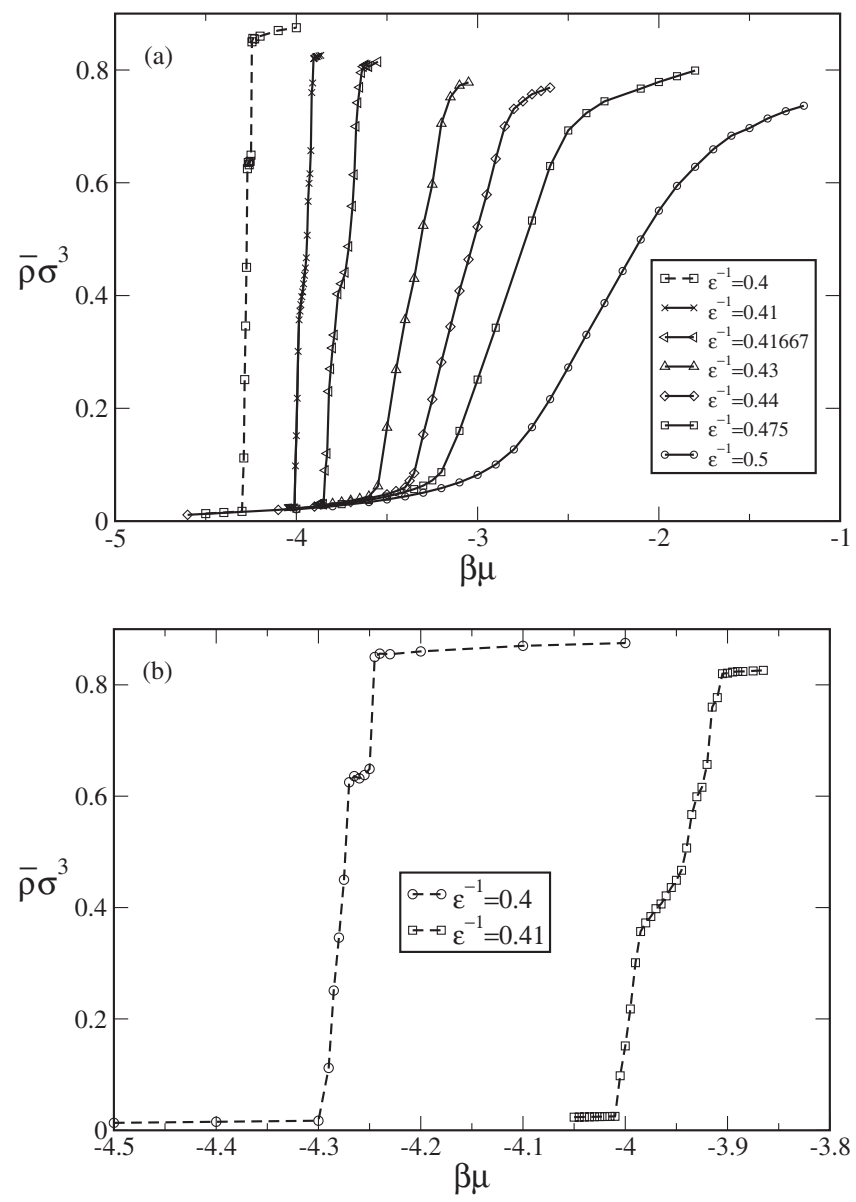

FIG. 2. (a) Measured forms of $\bar{\rho}(\mu)$ for $z_{1}=2, z_{2}=1, A=0.55$, and various $\epsilon^{-1}$. (b) An enlargement showing the data for $\epsilon^{-1}$ $=0.41$ and $\epsilon^{-1}=0.4$ as described in the text.

attractive strength for which nonhomogeneous states might be expected to occur. Guided by the DFT calculations reported in Sec. IV (which predict that for $A=0.55$, a $\lambda$ line appears for $0.54>\epsilon^{-1}>0.48$ ), we selected $\epsilon^{-1}=0.5$ as a suitable candidate. Subsequently it was found that smaller values of $\epsilon^{-1}$ were necessary to generate inhomogeneous phases.

For each value of $\epsilon^{-1}$ studied, the dependence of the average of the fluctuating particle number density, $\bar{\rho}$, on the applied chemical potential $\mu$ was measured. The resulting forms of $\bar{\rho}(\mu)$ are shown in Fig. 2(a). From this figure one observes that for the initial value $\epsilon^{-1}=0.5, \bar{\rho}(\mu)$ is essentially smooth, having a form reminiscent of a one-phase (supercritical) fluid. However, as $\epsilon^{-1}$ is reduced from this value, the gradient of the curves for moderate densities gradually increase in magnitude, indicating an increase in the fluid compressibility. Concomitantly, kinks in the curves start to develop at low and high density, which sharpen into discontinuities as $\epsilon^{-1}$ is further decreased. For $\epsilon^{-1} \lesssim 0.42$, these low and high density kinks are supplemented by additional ones at intermediate densities, as is apparent from the close-up of the data for $\epsilon^{-1}=0.41$ and $\epsilon^{-1}=0.4$ shown in Fig. 2(b).

We address first the phenomena underlying the appearance of the discontinuities in $\bar{\rho}(\mu)$ at low and high density. These arise from first-order phase transitions. In the low den-
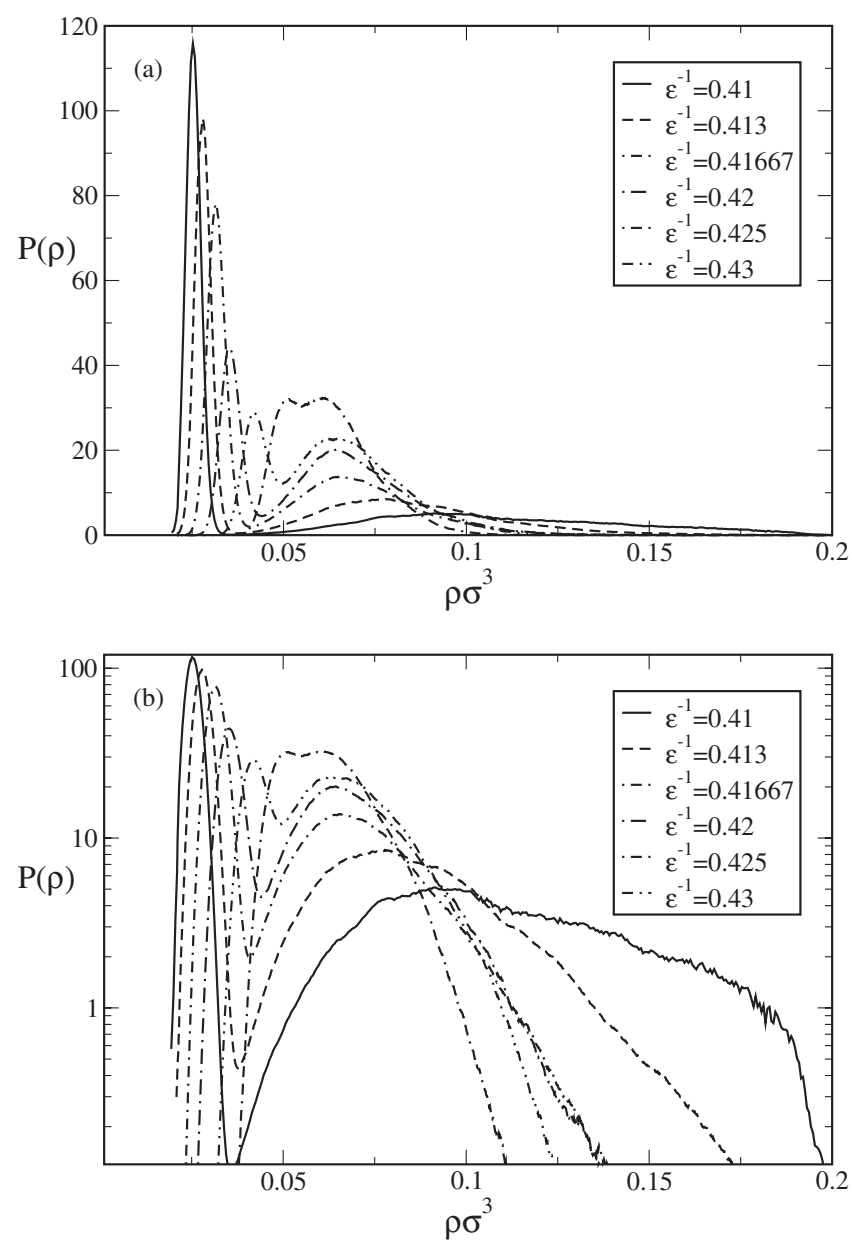

FIG. 3. (a) The distribution of the fluctuating number density for state points corresponding to the low density vapor-spherical cluster transition. (b) The same data plotted on a log scale.

sity case, the transition is between a vapor and an inhomogeneous phase composed of large spherical liquidlike clusters; in the high density case it is from a liquid to an inhomogeneous liquid phase containing large spherical vaporlike voids (i.e., bubbles). Discussion of the character of these inhomogeneous structures is deferred until Sec. III C.

Evidence for the existence of these phase transitions comes from the measured forms of the distribution of the fluctuating instantaneous number density $p(\rho)$ at the model parameters for which the kinks occur. For the low density transition, these distributions are shown in Fig. 3(a). One observes a two-peaked structure; the narrow peak at low densities corresponds to the vapor phase, while a much broader higher density peak corresponds to the cluster phase. At small values of $\epsilon^{-1}$ the two peaks are widely separated, the trough between them is deep [cf. the log scale of Fig. 3(b)], and the cluster peak is very broad [this latter feature reflects the large compressibility associated with the steepness of $\bar{\rho}(\mu)$ at these values of $\epsilon^{-1}$-recall Fig. 2(a)]. As $\epsilon^{-1}$ is increased, the two peaks approach one another and eventually merge into a single peak; this occurs at a value of $\epsilon^{-1}$ consistent with that at which the sharp low density kink in $\bar{\rho}(\mu)$ becomes smoothed out. 


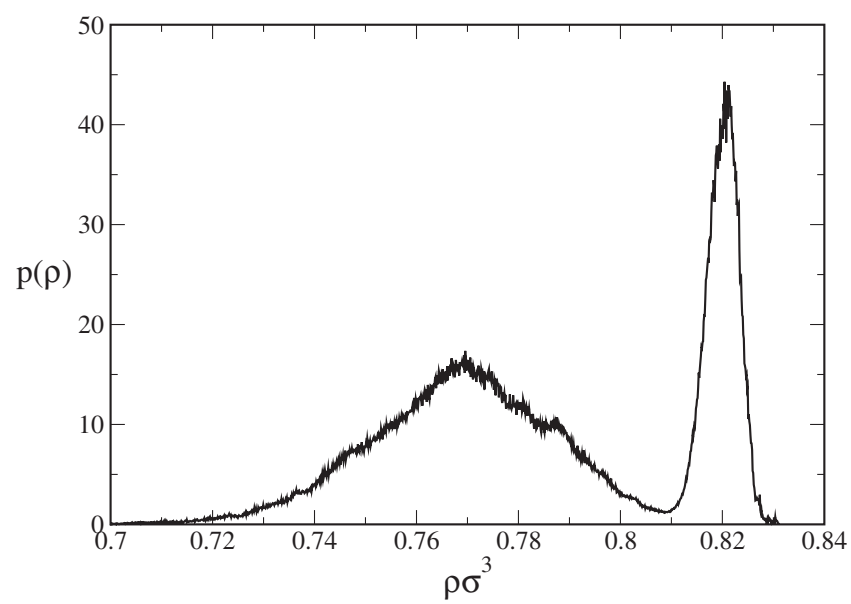

FIG. 4. The distribution of the fluctuating number density for the state point $\epsilon^{-1}=0.41, \beta \mu=-3.91$, lying on the high density spherical bubble-liquid transition.

The computational cost of performing long simulations in the region of the high density transition, verges on the prohibitive [55]. Consequently, we have been able to obtain the coexistence form of $p(\rho)$ for only a single value of $\epsilon^{-1}$, namely $\epsilon^{-1}=0.41$. This distribution is shown in Fig. 4 and exhibits a narrow liquidlike peak at high density together with a much broader peak at lower densities which corresponds to a liquid containing a large void, as will be discussed in Sec. III C. Again the parameters at which we find the double-peaked structure match those of the corresponding kink in $\bar{\rho}(\mu)$, which can therefore be taken as an alternative signature of the transition.

Unfortunately, it proved impossible to accurately quantify the number and energy densities of the cluster phases that coexist with the respective vapor and liquid phases. The problems are traceable to the large length scale associated with the typical cluster size, as illustrated in Fig. 5(a), which shows the measured density distribution $p(\rho)$ for two system sizes at a near-coexistence point located well inside the two-
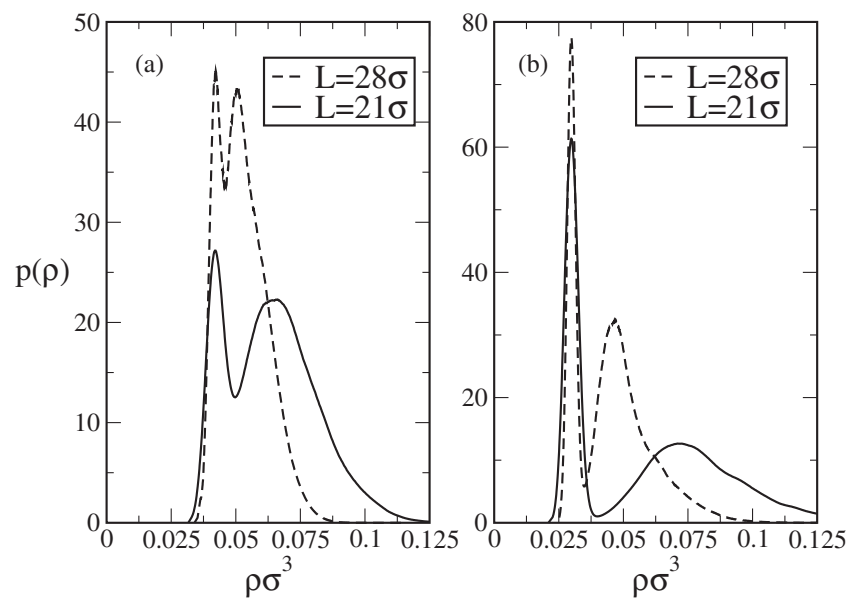

FIG. 5. Near-coexistence density distributions for (a) $\epsilon^{-1}$ $=0.415$ and (b) $\epsilon^{-1}=0.425$. In each case data is shown for two system sizes.

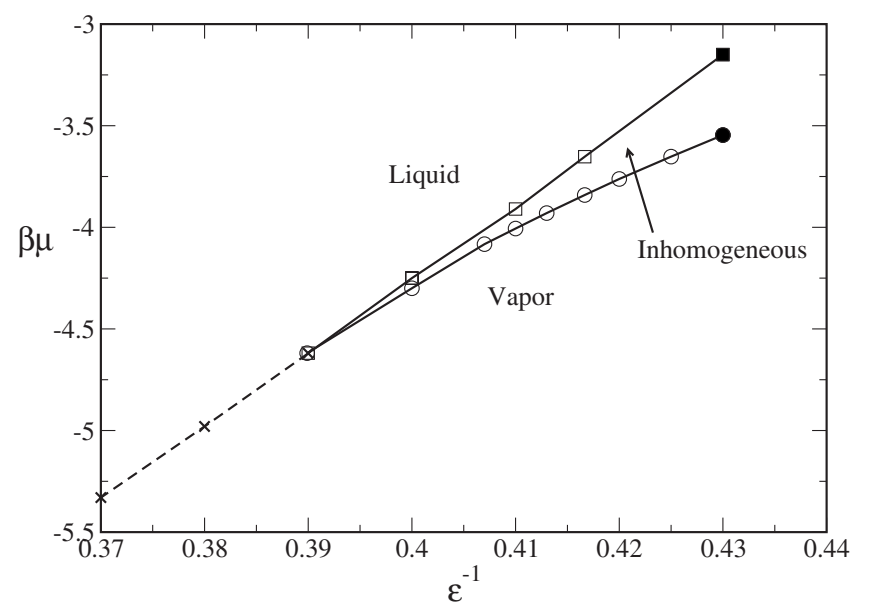

FIG. 6. Estimates of the phase diagram, as described in the text. Circles are the vapor-spherical cluster transition, squares are the spherical bubble-liquid transition. Crosses lie on the liquid-vapor coexistence line, lines merely guide the eye. Filled symbols locate the putative critical points. Uncertainties do not exceed the symbol sizes.

phase vapor-cluster region. From the figure, it is evident that while the position of the vapor phase peak is essentially system size independent, the density of the cluster peak shifts strongly to lower density as $L$ is increased. Thus, the system sizes attainable in this work fail to provide access to the thermodynamic limit for this phase. Analogous effects are found for the energy distribution (data not shown).

The low and high density first-order transition lines in $\epsilon$ - $\mu$ space are presented in Fig. 6. In this figure, the estimates of points on the low density transition line derive from the data of Fig. 3 by locating the values of $\mu$ for which $p(\rho)$ exhibits two peaks of approximately equal weight. Those for the high density transition derive both from the data of Fig. 4 for the case $\epsilon^{-1}=0.41$, and otherwise from the position of the high density kink in the $\bar{\rho}(\mu)$ curves. The loci of the two transition lines delimits a region within which inhomogeneous structures occur. For small $\epsilon^{-1} \simeq 0.39$, this region tapers down to a point, beyond which we were unable to stabilize inhomogeneous phases, instead finding a single transition from a vapor to a liquid. This suggests that a triple point occurs at $\epsilon^{-1}$ $\simeq 0.39$ below which standard vapor-liquid coexistence occurs. We have estimated the locus of a portion of the vaporliquid coexistence from the center of the hysteresis loop in $\epsilon$ - $\mu$ formed by traversing the transition from vapor to liquid and back again, and these estimates are also marked in Fig. 6. Owing to the great number of particles in the liquid phase, the considerable potential cutoff distance, and the substantial density difference between the liquid and vapor, it was not possible to link the phase spaces of coexisting vapor and liquid directly using biased sampling techniques, as was done for the low density (vapor-cluster) transition.

As regards the nature of the transitions for large $\epsilon^{-1}$, one observes from Fig. 3 that for the vapor-cluster transition, the two peaks in $p(\rho)$ coalesce at a value of $\epsilon^{-1}$ close to that at which the low density kink in $\bar{\rho}(\mu)$ disappears. It seems reasonable to assume that a critical point occurs in this region 
and that, by extension, the high density transition similarly end at a critical point near to where the high density kink in $\bar{\rho}(\mu)$ vanishes. However, accurately pinning down the respective critical point parameters, proved problematic. The source of the difficulty is, again, the large cluster length scale. Specifically, on increasing $\epsilon^{-1}$, the two peaks merge before any system size dependence of the vapor peak becomes apparent. This implies that (in contrast to, e.g., a liquid-vapor transition in a simple fluid [56]) the double peaked structure of $p(\rho)$ is lost before the thermal correlation length becomes comparable with the system size. Accordingly, the value of $\epsilon^{-1}$ at which the peaks merge in a finitesized system constitutes an underestimate of the true critical point value of $\epsilon^{-1}$ and can thus, strictly speaking, only provide a lower bound on the critical point value of $\epsilon^{-1}$ [57]. The existence of an additional large length scale for one of the phases severely complicates the implementation of standard finite-size methods for locating criticality, which are based on the assumption of a single dominant length scale in the near-critical region, namely the thermal correlation length. Nevertheless, for $\epsilon^{-1}>0.43$ the low and high density kinks in $\bar{\rho}(\mu)$ have disappeared, the curves are smooth, and we thus tentatively (and conservatively) assign $0.425 \leq \epsilon_{c}^{-1}$ $\leq 0.440$ for both the low density and high density transitions.

\section{Nature of the coexisting phases at the low and high density transitions}

In Sec. III B we mapped the boundary between the homogeneous and inhomogeneous phases. Here we ponder the character of the inhomogeneous phases in more detail. Figures 7 (a) and 7(b) show, for $\epsilon^{-1}=0.41$, typical configuration snapshots of the inhomogeneous phase which coexists with homogeneous vapor (liquid) at the low (high) density transitions respectively. For the low density transition, a vapor coexists with a phase containing an approximately spherical cluster, whose local density is liquidlike. For the high density transition, a dense liquid coexists with a phase containing a large spherical bubble of vapor.

The visual identification of clusters at the low density transition is corroborated by measurements of the radial distribution function $g(r)$ of the coexisting phases. Figure 8 displays the measured forms of $g(r)$ for the vapor and the coexisting spherical cluster phase at $\epsilon^{-1}=0.41$. One notes that while the vapor phase is, to a great extent, structureless [i.e., $g(r)$ effectively reaches its asymptotic value of unity for $r$ $\gtrsim 3 \sigma$ ], the cluster phase exhibits unusual features, namely a very pronounced enhancement in the value of $g(r)$ extending over a considerable length scale that is indicative of the cluster radius. Furthermore, as the inset shows, $g(r=10.5 \sigma)$ $\simeq 0.8$ - significantly less than unity. This latter feature heralds the onset of large-length-scale oscillations in $g(r)$ which have their origin in the intercluster correlations of the cluster fluid phase [32]. Further evidence for this assertion derives from our integral equation calculations to be presented in Sec. IV B.

The question naturally arises as to how the character of the spherical cluster phase alters as one tracks the transition line by varying $\epsilon^{-1}$. To answer it, in part at least, we have (a)
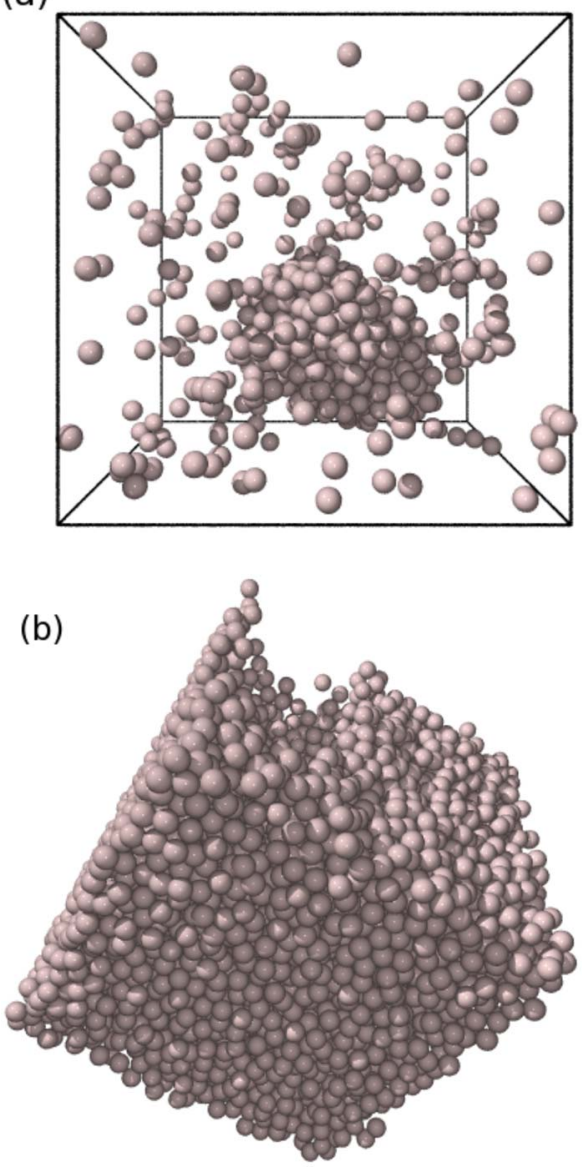

FIG. 7. (Color online) (a) A typical configuration of the spherical cluster phase which coexists with vapor (not shown) at the low density transition. (b) A typical configuration of the spherical vapor bubble phase that coexists with homogeneous liquid (not shown) at the high density transition.

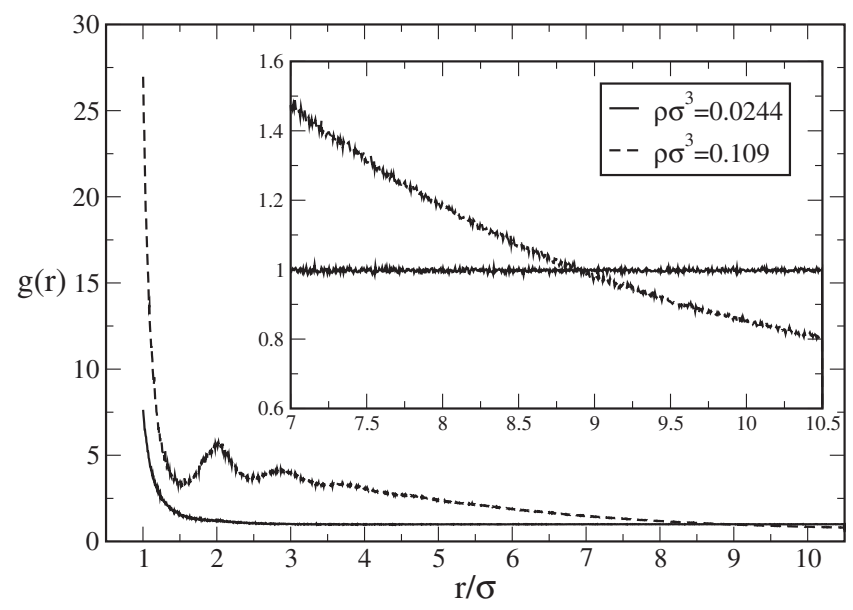

FIG. 8. The measured form of the radial distribution function $g(r)$ in both the vapor phase and the spherical cluster phase that coexist at the low density transition for $\epsilon^{-1}=0.41$. The inset shows an enlargement of the behavior at large $r$. 


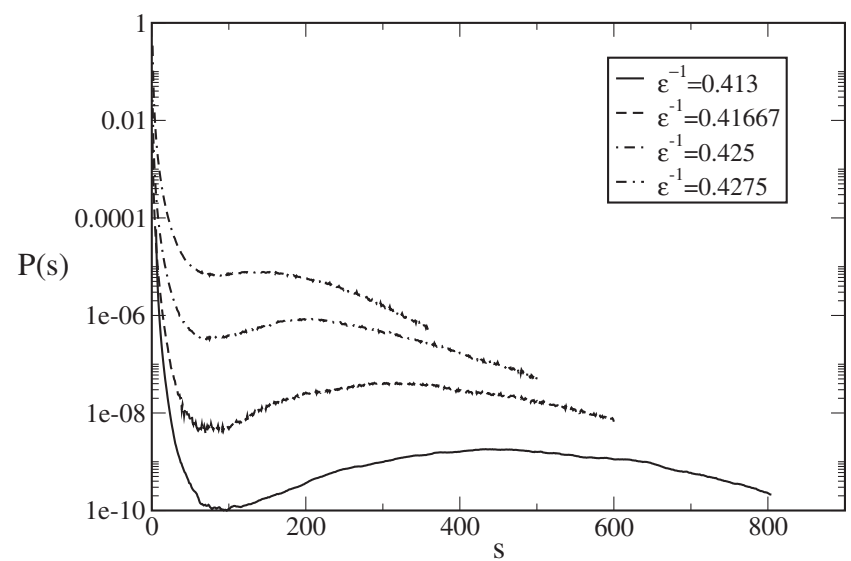

FIG. 9. Cluster mass distribution $p(s)$ (shown on a log scale) for a selection of points along the vapor-cluster transition. The curves have been shifted vertically to aid their distinguishability.

implemented a cluster identification algorithm [54] and used it to obtain the form of the cluster mass distribution $P(s)$ at the vapor-cluster transition, for a number of coexistence state points. The results (Fig. 9) show, in each instance, a double peaked distribution. The peak at low mass occurs at $s=1$, indicating that little or no clustering occurs in the vapor. The broad peak occurring at high mass indicates that the liquidlike clusters contain several hundred particles. The latter peak shifts strongly to greater masses with decreasing $\epsilon^{-1}$, showing that the typical radius of the clusters grows accordingly; this feature has important implications for finite-size effects, as we discuss below.

Finally in this section, we record that we have not attempted to characterize in any detail the properties of the inhomogeneous vapor-bubble phase occurring at the high density transition, due to the prohibitively large computational effort that this would entail.

\section{Further inhomogeneous states}

We consider next the structures that form as one traverses the region of inhomogeneous states separating the low and high density transitions. On increasing $\mu$ at constant $\epsilon^{-1}$ $=0.41$ from its coexistence value at the low density (vaporcluster) transition, visual inspection of typical configurations (Fig. 10) shows that the spherical clusters are replaced first by cylindrical clusters (which span the periodic system in one direction), and then, at still higher densities $\left(\bar{\rho} \sigma^{3} \simeq 0.2\right)$, by slablike structures (which span in two directions). In our simulations, one structure appeared to evolve smoothly into the next, and we could discern no clear signature of discontinuities in the gradient of the $\bar{\rho}(\mu)$ in this range of densities [cf. Fig. 2(b)]. Hence on this basis, there is no evidence for the existence of first-order phase transitions between spheres and cylinders or cylinders and slabs. However, given that the characteristic length scales of these structures is comparable with our system size, we cannot rule out that sharp transitions could become apparent for much larger system volumes.

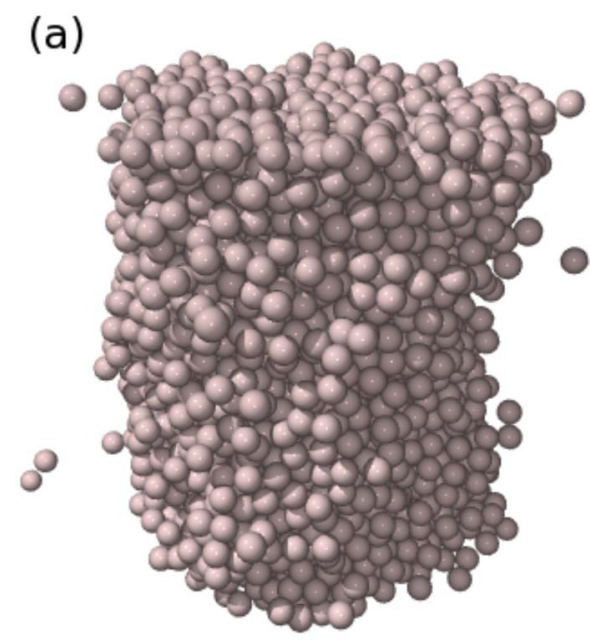

(b)

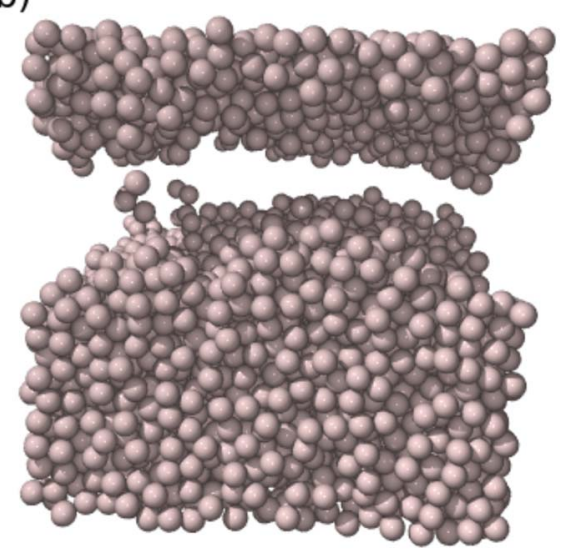

(c)

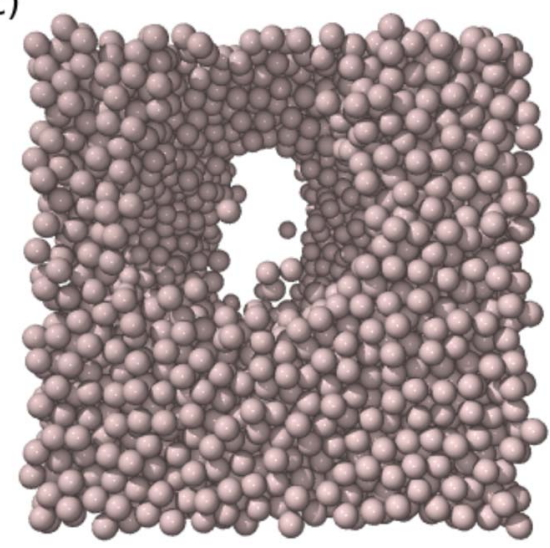

FIG. 10. (Color online) Snapshots of the typical sequence of finite system size-limited structures that were observed for $\epsilon^{-1}$ $=0.41$ when traversing the region of densities intermediate between the density of the spherical liquidlike cluster phase [which coexists with the vapor, cf. Fig. 7(a)], and the density of the spherical void phase [which coexists with the liquid, cf. Fig. 7(b)]. (a) A cylinder, (b) a slab, (c) a cylindrical void. See text for more details.

Moving to higher densities $\left(\bar{\rho} \sigma^{3} \geq 0.3\right)$, two kinks are visible in the $\bar{\rho}(\mu)$ curve for $\epsilon^{-1}=0.41$ [see Fig. 2(b)]. The first kink, appearing at a density of $\bar{\rho} \sigma^{3} \sim 0.37$, occurs in a region in which the configurations are slablike, and appears to relate 
to a single cuboidal slab being replaced by two parallel slabs. Accommodating an additional slab within the simulation box boosts the number of particles in each that are within range of the long ranged repulsions from particles occupying the other slab. Consequently it becomes energetically less favorable for the slabs to thicken as $\mu$ is increased further, leading to a decrease in the compressibility [and thus too, the gradient of $\bar{\rho}(\mu)]$. Accordingly, the kink at $\bar{\rho} \sigma^{3} \sim 0.37$ is something of a finite-size artifact. Visualization of configurations on either side of the second intermediate density kink (occurring at $\bar{\rho} \sigma^{3} \simeq 0.47$ ) reveals that this is associated with a transition from the parallel slabs of liquid just described, to a liquid containing large cylindrically shaped voids, or "bubbles" of vapor which span the system in one direction [Fig. $10(\mathrm{c})]$. A jump in the density at $\bar{\rho} \sigma^{3} \simeq 0.7$ corresponds to cylindrical voids being replaced by spherical voids.

Turning now to the data for $\epsilon^{-1}=0.4$, shown in Fig. 2(b), the kinks that form the plateau in $\bar{\rho}(\mu)$ at moderate densities have an origin distinct from that just described for the case $\epsilon^{-1}=0.41$. The configurations on either side of the first kink contain a single liquidlike slab. The plateau seems to develop because-as the density grows - the two surfaces of this single slab are forced together via the periodic boundaries until, at some point, the particles near one surface come within the range of the repulsive part of the potential of the particles at the other surface. Thus again, this feature would appear to be a finite-size artifact. The second kink marks an abrupt condensation of the slab into the homogeneous liquid phase.

The character of the inhomogeneous structures that occupy the region separating the pure phases helps to explain the dramatic increase in the compressibility [and hence the gradient of $\bar{\rho}(\mu)]$ on reduction of $\epsilon^{-1}$. This increase is (we believe) largely attributable to the increasing prevalence of slablike structures as $\epsilon^{-1}$ is reduced. Since the size of the spherical clusters that coexist with the pure phases grows with decreasing $\epsilon^{-1}$, they eventually approach that of the system size, at which point spheres or cylinders are no longer stable and are replaced by slabs. Since it costs little free energy to move a slab interface (except when the two interfaces interact via the periodic boundaries), the compressibility grows very large. We further note that for values of $\epsilon^{-1}$ $\lesssim 0.39$, i.e., below that of the triple point, one should expect such behavior since at densities intermediate between those of the stable coexisting vapor and liquid phases, slablike configurations dominate [58].

Summing up the findings of this section, we have presented evidence for a variety of inhomogeneous structures: spherical and cylindrical liquidlike clusters, single and multiple liquidlike slabs, cylindrical and spherical bubbles. However, the precise sequence of structures that occurs when traversing the inhomogeneous region at fixed $\epsilon^{-1}$ depends both on the value of $\epsilon^{-1}$ itself, and on the system size. This is perhaps not altogether surprising, given that the typical length scale of the inhomogeneous structures depends on $\epsilon^{-1}$ (cf. Fig. 9), and that for sufficiently small $\epsilon^{-1}$, this length scale can exceed the linear extent of the simulation box. Additionally, it should be mentioned that protracted relaxation times were encountered in the inhomogeneous region of the phase diagram. These arise because a local (i.e., single particle) update procedure requires many iterations in order to decorrelate a system having an inherently large length scale. Accordingly, great computational expenditure is necessary to ensure that the system attains the equilibrium structure for a given state point. In fact, we observed a certain amount of irreproducibility regarding the precise form of $\bar{\rho}(\mu)$ obtained on traversing the inhomogeneous region from vapor to liquid compared to the reverse path. The source of this irreproducibility is presumably traceable, in part at least, to the extended relaxation times.

\section{MEAN-FIELD AND INTEGRAL EQUATION THEORETICAL STUDIES}

\section{A. Mean-field theory of the fluid structure and thermodynamics}

In Ref. [32] the authors developed a mean-field DFT theory within the random phase approximation, for systems interacting via potentials of the form in Eq. (1). We will not describe in detail the theory here-instead referring interested readers to Ref. [32]. The key idea behind this approach (and indeed most other mean-field approaches) is to split the pair potential into two contributions: a reference part, $v_{r}(r)$ and the remainder or perturbation, $v_{p}(r)$, i.e., $v(r)=v_{r}(r)$ $+v_{p}(r)$. For the present system an obvious choice for the reference part is the hard-sphere potential, $v_{r}(r)=v_{\mathrm{hs}}(r)$, where

$$
v_{\mathrm{hs}}(r)= \begin{cases}\infty, & r \leq \sigma, \\ 0, & r>\sigma,\end{cases}
$$

and therefore,

$$
\beta v_{p}(r)= \begin{cases}-\epsilon+A, & r \leq \sigma \\ \beta v(r), & r>\sigma .\end{cases}
$$

Note that there is no unique choice for $v_{p}(r)$ in the range $r$ $\leq \sigma$, inside the hard core. The choice made in Eq. (3) is the same as that of Ref. [32].

The Helmholtz free energy of the system is approximated as follows [32]:

$$
F[\rho] \simeq F_{\mathrm{hs}}^{\mathrm{Ros}}[\rho]+\frac{1}{2} \int d \mathbf{r} \int d \mathbf{r}^{\prime} \rho(\mathbf{r}) \rho\left(\mathbf{r}^{\prime}\right) v_{p}\left(\left|\mathbf{r}-\mathbf{r}^{\prime}\right|\right),
$$

where $\rho(\mathbf{r})$ is the one-body density profile of the fluid and $F_{\mathrm{hs}}^{\mathrm{Ros}}[\rho]$ is the Rosenfeld approximation for the Helmholtz free energy of a hard-sphere fluid [59-61]. For the bulk fluid this amounts to the following (RPA) approximation for the pair direct correlation function $[32,44]$ :

$$
c(r ; \rho)=c_{\mathrm{hs}}^{\mathrm{PY}}(r ; \rho)-\beta v_{p}(r),
$$

where $c_{\mathrm{hs}}^{\mathrm{PY}}(r ; \rho)$ is the Percus-Yevick approximation for the hard-sphere direct pair correlation function for a bulk fluid of density $\rho[32,44]$.

For the potential parameters of concern in the present work $\left(z_{1}=2, z_{2}=1\right.$, and $\left.A=0.55\right)$, the mean-field theory of Ref. [32] yields the phase diagram displayed in Fig. 11. From 

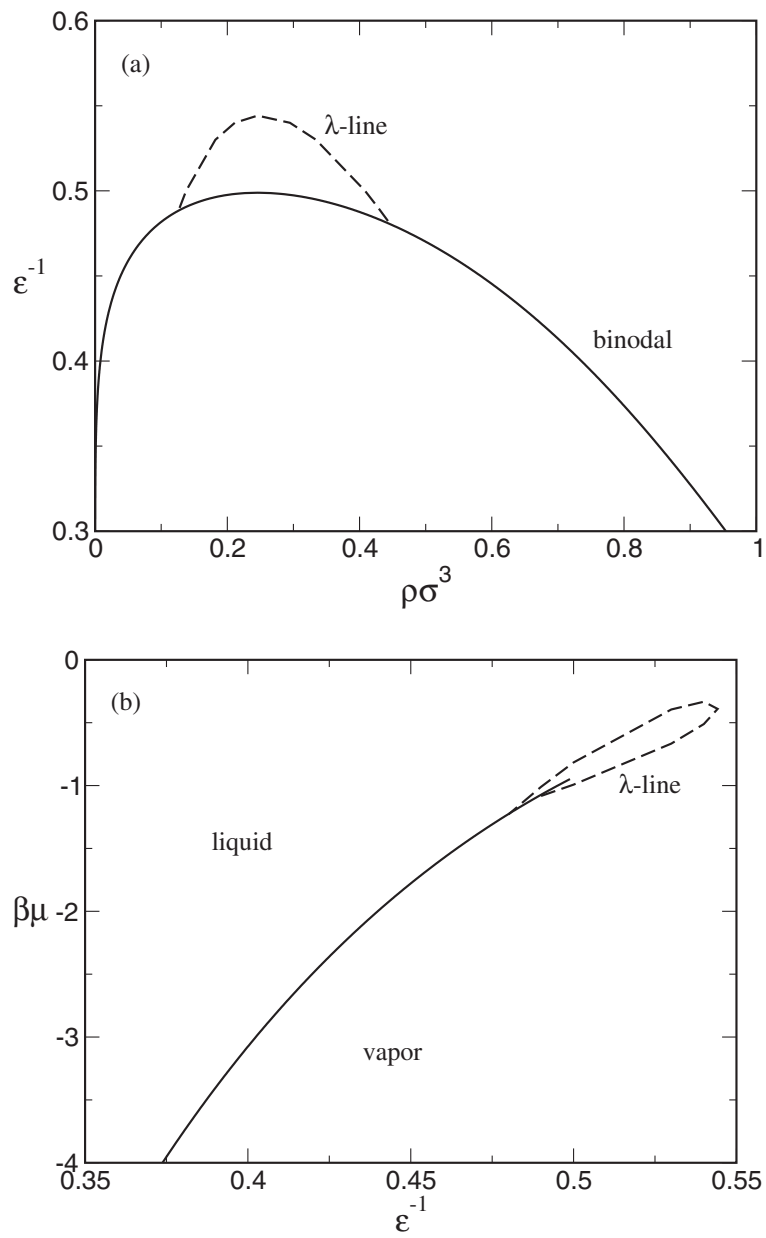

FIG. 11. Phase diagram for $z_{1}=2, z_{2}=1$, and $A=0.55$, obtained from the RPA DFT theory of Ref. [32]. The solid line is the binodal and the dashed line is the $\lambda$ line. (a) Plotted in the density $\rho$ versus $\epsilon^{-1}$ plane, (b) plotted in the chemical potential $\mu$ versus $\epsilon^{-1}$ plane.

this figure one sees that the theory predicts a $\lambda$ line enclosing the region of the phase diagram containing the liquid-vapor critical point. The $\lambda$ line is defined as the locus of points in the phase diagram for which the static structure factor $S(k)$ diverges at a particular wave number $k_{c} \neq 0$ [32]. We take the $\lambda$ line to indicate that the theory predicts a phase transition to a periodically modulated inhomogeneous phase. This phase preempts the standard liquid-vapor behavior that normally occurs in the subcritical region of a simple fluid. We note, however, that the topology of the $\lambda$ line and hence the region of inhomogeneous phase(s), is different from that obtained from the MC simulations-compare Figs. 6 and 11(b) - the $\lambda$ line suggests a closed loop around the inhomogeneous phase(s). This seems to be inconsistent with the simulation results of Sec. III, because the fluid of spherical clusters that we have identified is not periodically modulated. Thus while the RPA provides a reasonable approximation for the fluid structure and thermodynamics in regions of the phase diagram away from the $\lambda$ line [32], it would appear to be unreliable in its direct vicinity.

\section{B. Integral equation theories for the bulk fluid structure and thermodynamics}

The structure of a fluid may be characterized by the radial distribution function $g(r)=1+h(r)$, where $h(r)$ is the total correlation function, defined as the deviation of $g(r)$ from the ideal vapor result [44]. The radial distribution function is important for two main reasons. First, it yields-via a Fourier transform - the static structure factor

$$
S(k)=1+\rho \int d \mathbf{r}[g(r)-1] \exp (i \mathbf{k} \cdot \mathbf{r}),
$$

a quantity which, in principle, can be obtained in a scattering experiment. Second, $g(r)$ can be used to calculate thermodynamic quantities such as the internal energy and the pressure. For example, the pressure $P$ can be obtained via the virial equation [44]

$$
P=\rho k_{B} T-\frac{\rho^{2}}{6} \int d \mathbf{r} g(r) r \frac{d v(r)}{d r},
$$

while, the Helmholtz free energy may be obtained by thermodynamic integration. In practice, however, since $g(r)$ is only approximately known, the value obtained for the free energy is dependent upon the path of integration [62].

A key equation used to calculate $g(r)$ [or, equivalently $h(r)]$ is the Ornstein-Zernike equation [44]

$$
h(r)=c(r)+\rho \int d \mathbf{r}^{\prime} c\left(\left|\mathbf{r}-\mathbf{r}^{\prime}\right|\right) h\left(r^{\prime}\right),
$$

where $c(r)$ is the pair direct correlation function. To solve the $\mathrm{OZ}$ equation it must be supplemented by a closure relation. The exact closure reads [44]

$$
c(r)=-\beta v(r)+h(r)-\ln [1+h(r)]-b(r),
$$

where $-b(r)$ is the (unknown) bridge function. A simple approximation is to set $b(r)=0$. This is the HNC closure [44],

$$
c_{\mathrm{HNC}}(r)=-\beta v(r)+h(r)-\ln [1+h(r)] .
$$

We solve Eqs. (8) and (10) to obtain our approximation for $g(r)$. Another approximation we consider is the PY closure [44],

$$
c_{\mathrm{PY}}(r)=\{1-\exp [\beta v(r)]\}[1+h(r)],
$$

which is known to provide a good approximation for the structure and thermodynamics of a hard-sphere fluid at low and moderate densities.

Within the HNC approximation, the chemical potential may be obtained from the following expression:

$$
\beta \mu=\ln \left(\rho \Lambda^{3}\right)+\rho \int d \mathbf{r}\left(\frac{h(r)}{2}[h(r)-c(r)]-c(r)\right),
$$

where $\Lambda$ is the (irrelevant) thermal de Broglie wavelength. Coexistence between two phases occurs if they have equal temperature, equal pressure, and equal chemical potential. We employed Eqs. (7) and (12) to calculate the fluid pressure and chemical potential to find the coexisting densities within the HNC approximation. The results are displayed in Fig. 12. 


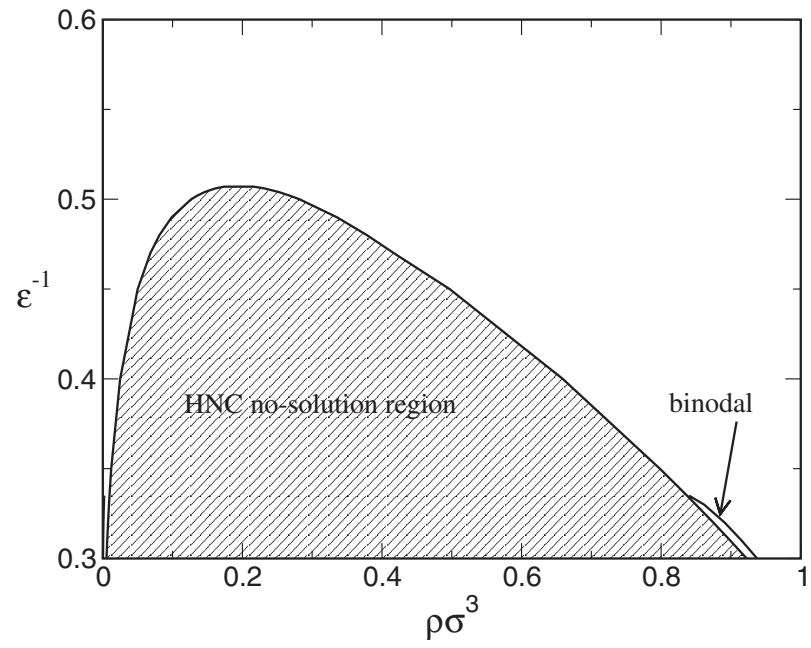

FIG. 12. The partial phase diagram in the $\epsilon^{-1}$ versus $\rho$ plane obtained from the HNC integral equation theory for $z_{1}=2, z_{2}=1$, and $A=0.55$. The shaded region denotes state points for which no solution could be found. Outside this region, at low and moderate densities, the HNC theory provides a good approximation for $g(r)$, when compared with MC data. We find that at low values of $\epsilon^{-1}$ the HNC predicts liquid-vapor coexistence.

Unfortunately, only a small portion of the coexistence binodal (at low values of $\epsilon^{-1}$ ) could be determined since for the $\mathrm{HNC}$ approximation there is a large region of the phase diagram in which there is no solution to the $\mathrm{OZ}$ equation. This region encompasses state points where we would expect (on the basis of the simulation) to find inhomogeneous phase(s) - see Fig. 12. We note that it is not unknown for the HNC approximation to exhibit regions of no solutions [63-66]. Indeed, one can obtain the HNC closure (10) as the Euler-Lagrange equation from an associated free energy functional and it can be shown that the lack of HNC solutions is due to the loss of convexity in this free energy functional $[65,66]$.

We have also applied a modified HNC (MHNC) closure in which we invoke a simple approximation for the bridge function in Eq. (9), chosen to be the PY expression for the hard-sphere fluid bridge function at the same density $\rho$. We found that this MHNC approximation failed to converge for the same state points as the HNC approximation. We did not attempt to implement any other more sophisticated MHNC approximations [62].

Applying the PY closure (11) to the OZ equation (8) gives some (perhaps) surprising results: As with the HNC, there are regions of the phase diagram where we were unable to obtain a solution. However, their location is different to that found for the HNC-compare Figs. 12 and 13. The theory also seems to succeed in capturing some key aspects of the cluster transition. Specifically, if for some constant $\epsilon^{-1}$ $\lesssim 0.48$, one calculates the PY approximation for $g(r)$ along a path of increasing density, starting from very low densities, one finds at some $\rho$ that a discontinuous jump occurs in the solution for $g(r)$ - see, for example, the results in Figs. 14 and 15. The locus of points in the phase diagram at which $g(r)$ jumps in this way is displayed in Fig. 13. Note also, that if one traverses the reverse path, one can follow the high

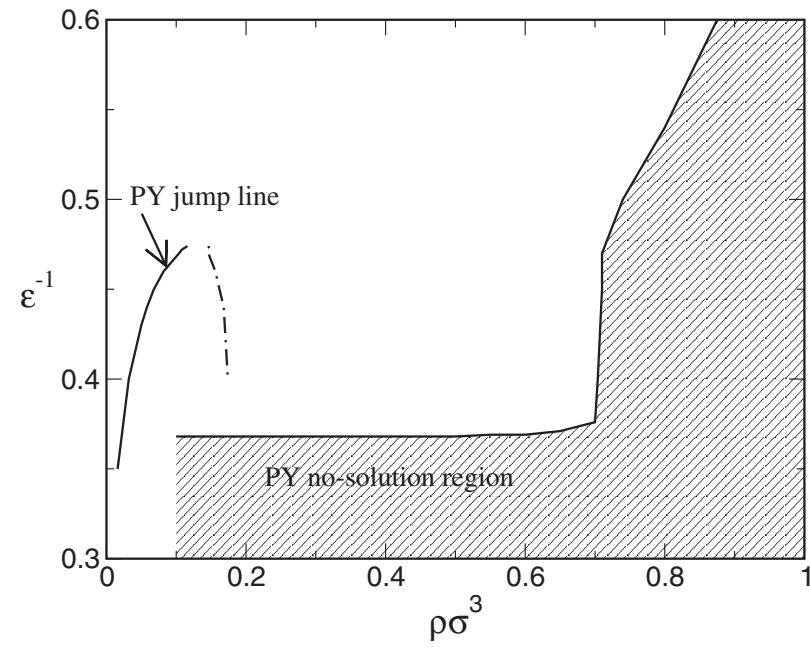

FIG. 13. The partial phase diagram in the $\epsilon^{-1}$ versus $\rho$ plane as obtained by the PY integral equation theory. The shaded region denotes state points for which no solution could be found. The solid line indicates the line in the phase diagram at which there is a discontinuous change in $g(r)$ —compare with Figs. 14 and 15. This line is close to the vapor-cluster phase transition in our MC simulations. The dotted-dashed line indicates the locus of state points at which the pressure [obtained via Eq. (7)] equals that in the vapor phase.

density branch of solutions to the PY displaying the clustering to very low densities. The low density solutions on this branch are particularly striking. The location in the phase diagram of the jump line is close to where the transition to a cluster phase occurs, as determined from our MC simulations.

In Fig. 16 we display $|h(r)|$ obtained from the PY theory for the state point $\epsilon^{-1}=0.41$ and $\rho \sigma^{3}=0.220$ (see also Fig.

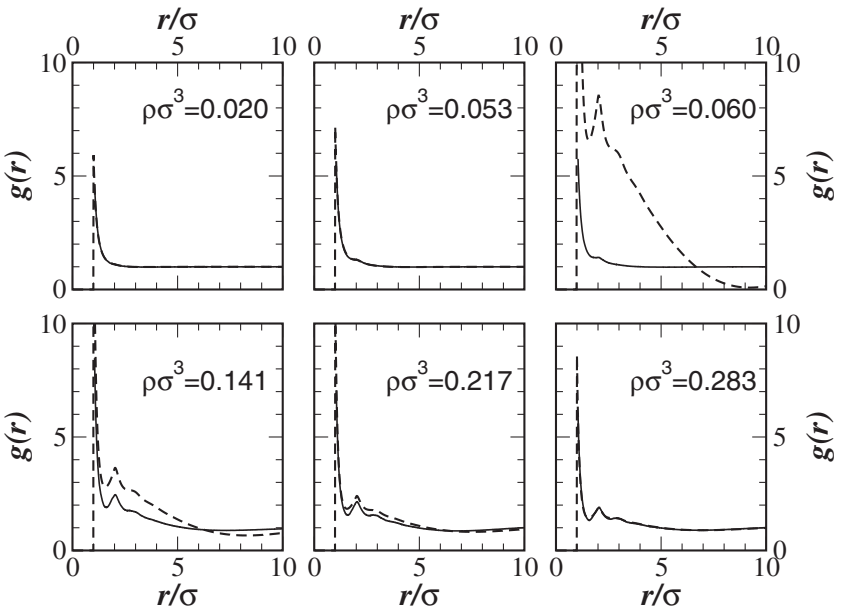

FIG. 14. The radial distribution function, $g(r)$, obtained from simulation (solid lines) and the PY theory (dashed lines) for $\epsilon^{-1}$ $=0.44$ at the particular selection of densities indicated. Note that at $\rho \sigma^{3}=0.058$, the PY theory predicts a dramatic jump discontinuity in $g(r)$. An increase in the magnitude of the first and second maxima also occurs in the MC simulation results at a slightly higher density, but it is much less pronounced than that predicted by the PY theory and does not appear to be discontinuous for this value of $\epsilon^{-1}$. 

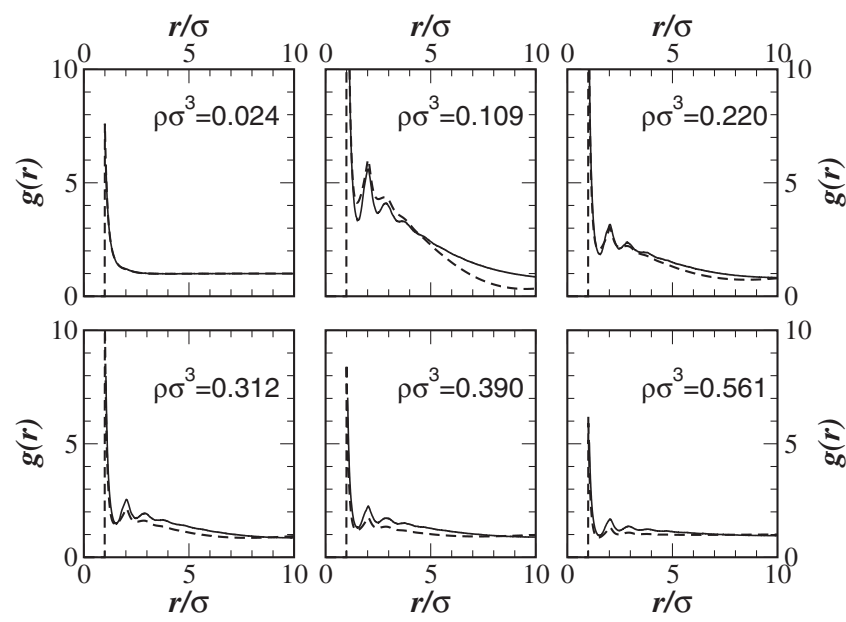

FIG. 15. The radial distribution function, $g(r)$, obtained from simulation (solid lines) and the PY theory (dashed lines) for $\epsilon^{-1}$ $=0.41$ at the particular selection of densities indicated. Note that in both the simulations and theory, a jump discontinuity occurs in $g(r)$ between $\rho \sigma^{3}=0.024$ and $\rho \sigma^{3}=0.109$. Within the PY theory, this jump occurs at $\rho \sigma^{3}=0.04$.

15). The logarithmic vertical axis allows inspection of the long wavelength oscillatory decay of this correlation function, arising from intercluster correlations. A precursor to this oscillatory behavior is visible in our simulation results (Fig. 8). We note that the amplitude of the oscillations decays rather slowly indicating very long ranged correlations in the cluster phase.

We have attempted to calculate the binodal of the vaporcluster transition from PY theory. Initially we sought to perform thermodynamic integration of the pressure [as obtained from the virial (7)] to acquire the Helmholtz free energy [62] and thence the coexisting state points. However, we were unsuccessful in this endeavor due to insufficient selfconsistency within the theory. As an alternative, more approximate, approach we have performed the following: (i) We assumed that the jump line obtained from the PY theory (see Fig. 13) is roughly the coexistence curve for the vapor

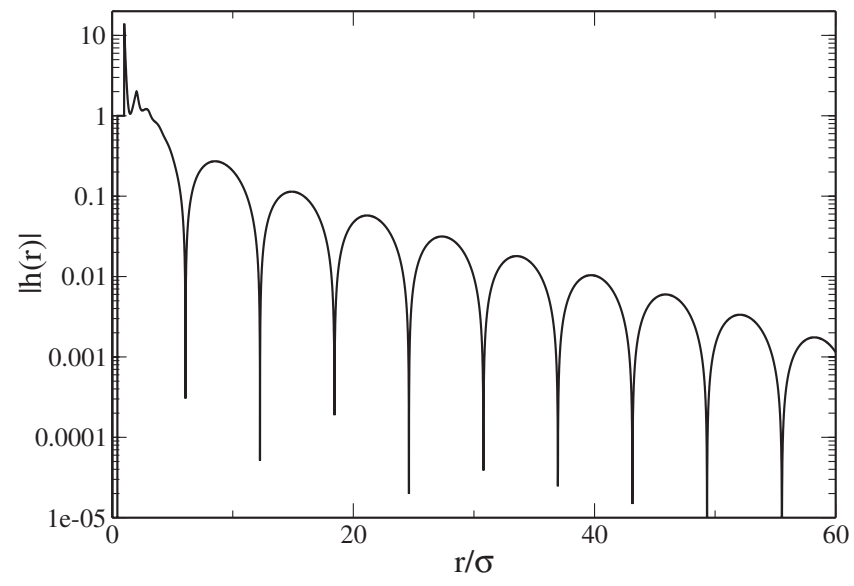

FIG. 16. The modulus of the correlation function $h(r)$ obtained from the PY theory for $\epsilon^{-1}=0.41$ and $\rho \sigma^{3}=0.220$.

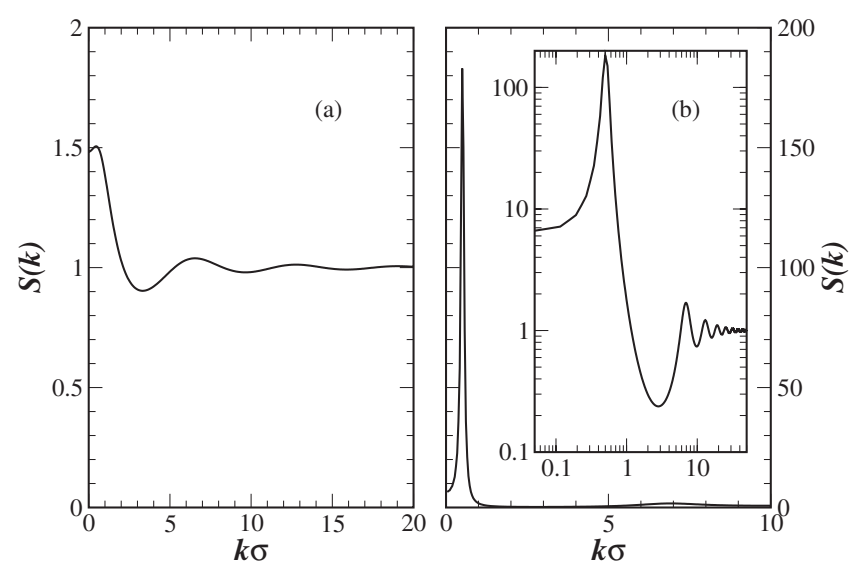

FIG. 17. The static structure factor, $S(k)$, obtained for the PY theory for the state points $A=0.55, \epsilon^{-1}=0.41$ and for densities (a) $\rho \sigma^{3}=0.024$, (b) $\rho \sigma^{3}=0.220$. For both of these state points the agreement between the PY results and the MC simulations for $g(r)$ is good-see Fig. 15. The inset to (b), shows $S(k)$ on a logarithmic scale.

phase-our MC simulation data supports this assumption. (ii) To find the coexisting cluster phase density, we sought the cluster phase state point having the same value of $\epsilon^{-1}$ and pressure [obtained via Eq. (7)] as the vapor phase at densities just below the jump line. This approach yields the dotteddashed line in Fig. 13. Similar results can be obtained from an alternative approach, which is to plot the pressure obtained from the virial equation (7) as a function of density for fixed $\epsilon^{-1}<0.477$; this produces a curve exhibiting a van der Waals loop (as well as a discontinuity at the jump line). Ignoring the discontinuity, one can perform the equal areas construction in order to obtain coexisting state points.

In Fig. 17 we display the static structure factor $S(k)$ obtained from the PY theory for two state points on either side of the vapor-cluster phase transition line. For these state points the PY result for $g(r)$ agrees well with our MC simulation result—see Fig. 15. $S(k)$ displays a peak at small wave vector $0<k_{c} \ll 2 \pi / \sigma$ for states on both sides of the transition line. This peak is known to be a signature of clustering. However, in the cluster phase the peak height is several orders of magnitude greater than in the vapor phase.

Figure 18 shows the peak (maximal) value of $S(k)$ obtained from the PY theory as a function of the fluid density $\rho$, for a selection of values of $\epsilon^{-1}$. At low $\rho, S(k) \simeq 1 \forall k$. As the density is increased, the peak that grows the fastest, is that at $k=k_{c}$, i.e., the curves in Fig. 18 represent the dependence of $S\left(k_{c}\right)$ on $\rho$ in this regime. For values of $\epsilon^{-1}<0.48$, a jump occurs in $S\left(k_{c}\right)$ at the density of the vapor-cluster transition. Underlying this jump in the peak height is a wholesale discontinuous change in the entire form of $S(k)$. As we have previously shown [32], the value of the structure factor at $k=0$ is roughly proportional to its value at $k=k_{c}$, and consequently, $S(k=0)$ also jumps at the transition point. Given, however, that $S(0)=\rho k_{B} T \chi_{T}$, where $\chi_{T}$ is the isothermal compressibility, this in turn implies that a jump discontinuity occurs in $\chi_{T}$ at the transition density, in accordance with the simulation results. 


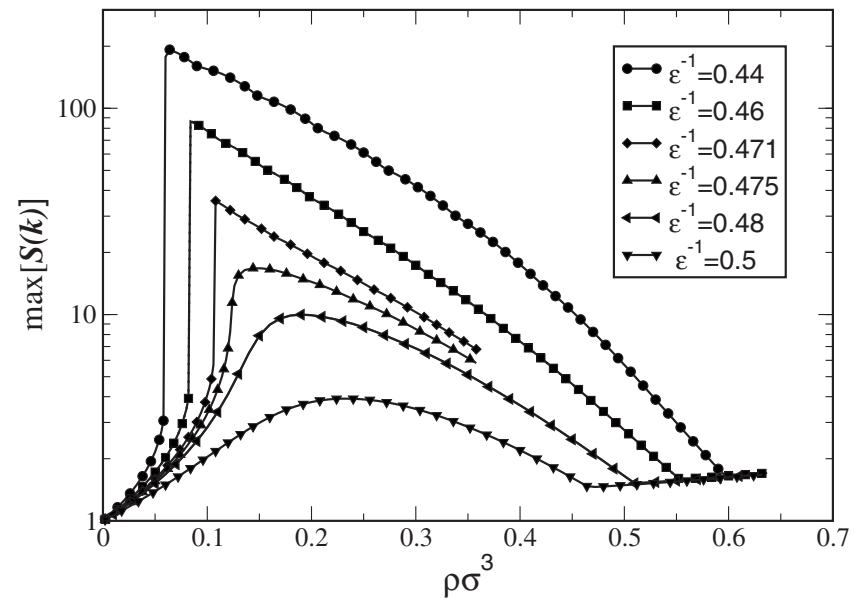

FIG. 18. The behavior of the maximal value of the static structure factor $S(k)$ as a function of $\rho$, obtained within the PY theory for a number of different values of $\epsilon^{-1}$, as described in the text.

As the density is increased past the value at which $S(k)$ jumps as a whole, the value of $S\left(k_{c}\right)$, i.e., the peak height decreases. Eventually, at some higher density (the precise value depending on the particular value of $\epsilon^{-1}$ ), the maximal value of $S(k)$ no longer occurs at $k=k_{c} \neq 0$, but instead at $k$ $\simeq 2 \pi / \sigma$, the next maximum in $S(k)$. This next maximum originates from the hard-sphere correlations in the fluid, and the crossover in maximal value from one peak in $S(k)$ to the other, accounts for the higher density discontinuity in the gradient of the curves displayed in Fig. 18. For example, for the case when $\epsilon^{-1}=0.44$, this occurs at $\rho \sigma^{3} \simeq 0.6$.

In Fig. 19 we display the direct pair correlation function for the state point $A=0.55, \epsilon^{-1}=0.41$ and density $\rho \sigma^{3}$ $=0.220$. For this state point the PY approximation for $g(r)$ is in good agreement with the results from our $\mathrm{MC}$ simulations-see Fig. 15. Note that for $1<r / \sigma<1.3$, $c(r) \gg-\beta v(r)$. We believe it is for this reason that the MSA closure to the $\mathrm{OZ}$ equation,

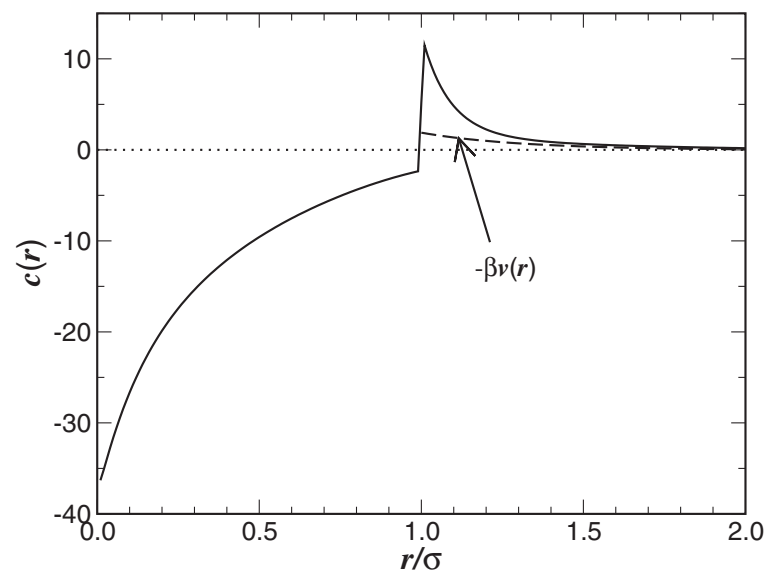

FIG. 19. The direct pair correlation function $c(r)$ obtained from the PY theory for the parameters $A=0.55$ and $\epsilon^{-1}=0.41$ and density $\rho \sigma^{3}=0.220$. The dashed line is a plot of $-\beta v(r)$. For the corresponding $g(r)$, see Fig. 15, and for the corresponding $S(k)$, see Fig. 17.

$$
\begin{gathered}
h(r)=-1, \quad r<\sigma, \\
c(r)=-\beta v(r), \quad r>\sigma,
\end{gathered}
$$

completely fails to describe the fluid structure in the region of the phase diagram near the vapor-cluster phase transition-the MSA approximation forces $c(r)$ to be much smaller than it is in reality for $1<r / \sigma<1.3$. We believe that it is also for this reason that the RPA closure, Eq. (5), is unreliable in the vicinity of the vapor-cluster transition.

\section{DISCUSSION}

In summary, we have studied the phase behavior of a system of particles which interact via a SALR potential, as a function of the strength of the short ranged attraction, for a fixed strength of the long ranged repulsion. The effect of the repulsion is to substitute the liquid-vapor critical point and a portion of the associated liquid-vapor transition line, with two first-order phase transitions: one from the vapor to a fluid of spherical liquidlike clusters, and the other from the liquid to a fluid of spherical voids. Each of these phases is highly compressible compared to the respective homogeneous phase with which it coexists. At low temperature, the two transition lines intersect one another and the vapor-liquid transition line at a triple point, while at high temperatures, they appear to terminate at distinct critical points. Although SCOZA, HRT and most of the standard integral equation theories are unable to describe the new transitions, somewhat surprisingly the Percus-Yevick approximation does succeed in capturing the vapor-cluster phase transition, as well as key aspects of the structure of the cluster fluid.

Owing to the high computational cost of the current study (which consumed 2-3 years of CPU time on a $3 \mathrm{GHz}$ processor), we have obtained the phase behavior only for a single value of the strength of the repulsion namely $A$ $=0.55$. Consequently, it is difficult to comment authoritatively on the topology of the phase diagram in the full space of $\mu, \epsilon$, and $A$. Nevertheless, it is worthy of note that the phase diagram of Fig. 6 is largely consistent with a cut at constant $A$ through a phase diagram originally obtained by Barbosa in a mean-field study of an Ising model having isotropic competing interactions [67]. Figure 20(a) recasts Barbosa's phase diagram in terms appropriate for the present fluid model. One sees from the figure that as $A$ is increased from zero, the liquid-vapor critical point shifts to lower $\epsilon^{-1}$ in accordance with SCOZA and HRT studies [31] of SALR potentials. At some value of $A=A_{L}$-corresponding to a Lifshitz point-inhomogeneous phases start to occur and two sheets (or wings) of first-order phase transitions emerge from a triple line to delineate a region of inhomogeneous states. This region is capped off at high $\epsilon^{-1}$ by a surface of continuous transitions. A cut through the phase diagram at constant $A \gtrsim A_{L}$ has the form shown in Fig. 20(b), which is qualitatively very similar to our Fig. 6, except that we have not, as yet, been able to identify a line of continuous transitions.

A phase diagram of the form Fig. 20(a) might shed light on the observation by Pini et al. of an anomalously large region of high compressibility around the critical point [31]. 

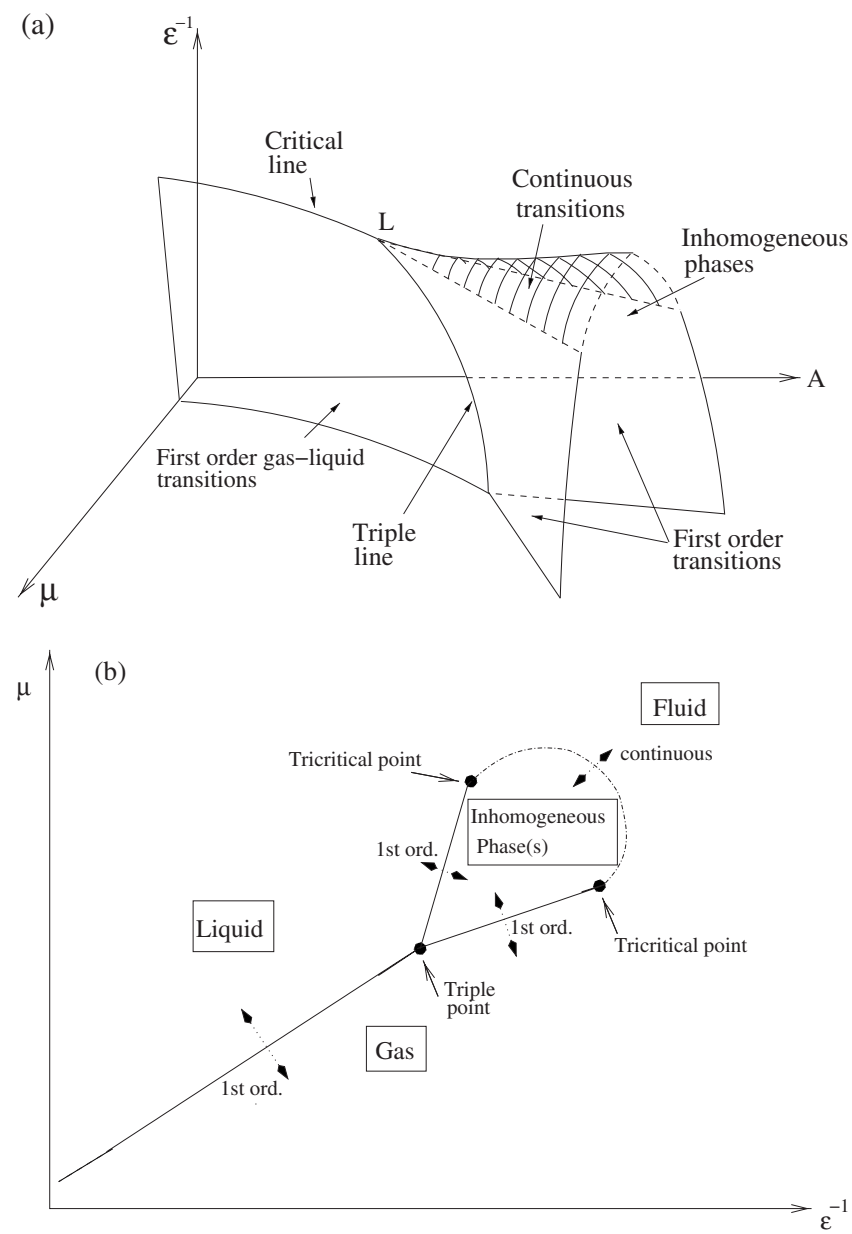

FIG. 20. (a) A schematic representation of a possible form of the full phase diagram for the model of Eq. (1), adapted from Ref. [67] and described in the text. (b) A cut at constant $A \gtrsim A_{L}$ through the phase diagram of (a).

If the strength of the repulsion is such that $A \lesssim A_{L}$, then the system will exhibit a liquid-vapor critical point, but will be located (in the full phase diagram) close to the Lifshitz point. As we have seen in the present work, the inhomogeneous states that form for $A>A_{L}$ are highly compressible compared to the vapor or the liquid, and even though they are not stable for $A \lesssim A_{L}$, they would be expected to have an influence on the free energy landscape. This could account for the anomalously large region of high compressibility.
As described in Sec. III D, when traversing the inhomogeneous region separating the low and high density transitions, a variety of finite-size limited (spanning) structures were observed depending on the value of $\epsilon$ and the system size. Evidence was found for cylinders and slabs, as well as cylindrical voids. It is interesting to note that a similar sequence of inhomogeneous structures has also been reported in simulations of the subcritical Lennard-Jones fluid using periodic boundary conditions [68]. There the observed structures occurred for values of the density intermediate between the coexisting stable vapor and liquid phases, and were metastable with respect to these phases. It therefore seems that the effect of adding a long ranged repulsion to a purely attractive fluid, is to stabilize these structures with respect to the homogeneous phases. We further note that while the clusters that occur at the low and high density transitions have a length scale smaller than our system size (at least for larger $\epsilon^{-1}$ ), and are therefore expected to persist in the thermodynamic limit, our system sizes are too limited to make definitive statements regarding the situation at moderate densities. Here it seems more likely that modulated structures occur, the wavelength of which exceeds our linear system sizes.

With regard to the prospects for extensions to the present work, we were not able to satisfactorily address the question of the nature of the putative critical points which terminate the vapor-cluster and liquid-cluster transitions. To do so will require the identification of the appropriate order parameter for the transitions, and a reformulation of finite-size scaling methodologies to take account of the large cluster length scale [69]. Once this is achieved, the question as to whether a line of continuous transitions really does link the (tri)critical points [cf. Fig. 20(b)] could be tackled. As for future integral equation studies, another closure approximation for the OZ equation, in common employ, is the Rogers-Young (RY) closure [62,70]. This interpolates between the PY and HNC theories in such a way as to enforce consistency between the virial and the compressibility routes to the thermodynamics. Since the PY theory is able to describe the vaporcluster phase transition, in contrast to the HNC, it would be interesting to see how the RY closure fares in this respect.

\section{ACKNOWLEDGMENTS}

The authors have benefited from helpful discussions with R. Evans, D. Pini, and L. Reatto. One of the authors (A.J.A.) gratefully acknowledges support from RCUK.
[1] For a review see G. Malescio, J. Phys.: Condens. Matter 19, 073101 (2007).

[2] E. A. Jagla, J. Chem. Phys. 111, 8980 (1999).

[3] N. B. Wilding and J. E. Magee, Phys. Rev. E 66, 031509 (2002).

[4] H. M. Gibson and N. B. Wilding, Phys. Rev. E 73, 061507 (2006).

[5] B. M. Mladek, D. Gottwald, G. Kahl, M. Neumann, and C. N. Likos, Phys. Rev. Lett. 96, 045701 (2006).
[6] C. N. Likos, N. Hoffmann, H. Löwen, and A. A. Louis, J. Phys.: Condens. Matter 14, 7681 (2002).

[7] G. Malescio and G. Pellicane, Nat. Mater. 2, 97 (2003).

[8] R. P. Sear and D. Frenkel, Phys. Rev. Lett. 90, 195701 (2003).

[9] P. J. Camp, Phys. Rev. E 68, 061506 (2003).

[10] A. Stradner, H. Sedgwick, F. Cardinaux, W. C. K. Poon, S. U. Egelhaaf, and P. Schurtenberger, Nature (London) 432, 492 (2004)

[11] H. Sedgwick, S. U. Egelhaaf, and W. C. K. Poon, J. Phys.: 
Condens. Matter 16, S4913 (2004).

[12] A. I. Campbell, V. J. Anderson, J. S. van Duijneveldt, and P. Bartlett, Phys. Rev. Lett. 94, 208301 (2005).

[13] R. Sanchez and P. Bartlett, J. Phys.: Condens. Matter 17, S3551 (2005).

[14] J.-L. Barrat and J.-P. Hansen, Basic Concepts for Simple and Complex Liquids (Cambridge University Press, Cambridge, 2003).

[15] F. Bordi, C. Cametti, S. Sennato, and M. Diociaiuti, Biophys. J. 91, 1513 (2006).

[16] F. Lo Verso, C. N. Likos, and L. Reatto, Prog. Colloid Polym. Sci. 133, 78 (2006).

[17] J. Chakrabarti, S. Chakrabarti, and H. Löwen, J. Phys.: Condens. Matter 18, L81 (2006).

[18] F. Ghezzi and J. C. Earnshaw, J. Phys.: Condens. Matter 9, L517 (1997).

[19] R. P. Sear, S.-W. Chung, G. Markovich, W. M. Gelbart, and J. R. Heath, Phys. Rev. E 59, R6255 (1999).

[20] A. Imperio and L. Reatto, J. Phys.: Condens. Matter 16, S3769 (2004).

[21] A. Imperio and L. Reatto, J. Chem. Phys. 124, 164712 (2006).

[22] D. Andelman, F. Brochard, and J.-F. Joanny, J. Chem. Phys. 86, 3673 (1986).

[23] G. F. Kendrick, T. J. Sluckin, and M. J. Grimson, Europhys. Lett. 6, 567 (1988).

[24] M. Seul and D. Andelman, Science 267, 476 (1995).

[25] Z. Nussinov, J. Rudnick, S. A. Kivelson, and L. N. Chayes, Phys. Rev. Lett. 83, 472 (1999).

[26] C. B. Muratov, Phys. Rev. E 66, 066108 (2002).

[27] M. Tarzia and A. Coniglio, Phys. Rev. E 75, 011410 (2007).

[28] R. P. Sear and W. M. Gelbart, J. Chem. Phys. 110, 4582 (1999).

[29] J. Groenewold and W. K. Kegel, J. Phys. Chem. B 105, 11702 (2001).

[30] J. Groenewold and W. K. Kegel, J. Phys.: Condens. Matter 16, S4877 (2004).

[31] D. Pini, G. Jialin, A. Parola, and L. Reatto, Chem. Phys. Lett. 372, 209 (2000).

[32] A. J. Archer, D. Pini, R. Evans, and L. Reatto, J. Chem. Phys. 126, 014104 (2007).

[33] J. Wu, Y. Liu, W.-R. Chen, J. Cao, and S.-H. Chen, Phys. Rev. E 70, 050401(R) (2004).

[34] Y. Liu, W.-R. Chen, and S.-H. Chen, J. Chem. Phys. 122, 044507 (2005).

[35] M. Broccio, D. Costa, Y. Liu, and S.-H. Chen, J. Chem. Phys. 124, 084501 (2006).

[36] F. Sciortino, S. Mossa, E. Zaccarelli, and P. Tartaglia, Phys. Rev. Lett. 93, 055701 (2004).

[37] S. Mossa, F. Sciortino, P. Tartaglia, and E. Zaccarelli, Langmuir 20, 10756 (2004).

[38] F. Sciortino, P. Tartaglia, and E. Zaccarelli, J. Phys. Chem. B 109, 21942 (2005).

[39] A. de Candia, E. Del Gado, A. Fierro, N. Sator, M. Tarzia, and
A. Coniglio, Phys. Rev. E 74, 010403(R) (2006).

[40] P. Charbonneau and D. R. Reichman, Phys. Rev. E 75, 011507 (2007).

[41] A. J. Archer, C. N. Likos, and R. Evans, J. Phys.: Condens. Matter 16, L29 (2004).

[42] G. Stell, J. Stat. Phys. 78, 197 (1995).

[43] A. Ciach, W. T. Góźdź, and R. Evans, J. Chem. Phys. 118, 3702 (2003).

[44] J.-P. Hansen and I. R. McDonald, Theory of Simple Liquids (Academic, London, 2006).

[45] E. Canessa, M. J. Grimson, and M. Silbert, Mol. Phys. 67, 1153 (1989).

[46] M. Silbert, E. Canessa, M. J. Grimson, and O. H. Scalise, J. Phys.: Condens. Matter 11, 10119 (1999).

[47] A. Parola, D. Pini, and L. Reatto, J. Phys.: Condens. Matter 18, S2305 (2006).

[48] P. J. Lu, J. C. Conrad, H. M. Wyss, A. B. Schofield, and D. A. Weitz, Phys. Rev. Lett. 96, 028306 (2006).

[49] M. H. J. Hagen and D. Frenkel, J. Chem. Phys. 101, 4093 (1994).

[50] D. Frenkel and B. Smit, Understanding Molecular Simulation (Academic, San Diego, 2002).

[51] B. A. Berg and T. Neuhaus, Phys. Rev. Lett. 68, 9 (1992).

[52] A. M. Ferrenberg and R. H. Swendsen, Phys. Rev. Lett. 63, 1195 (1989).

[53] N. B. Wilding, Am. J. Phys. 69, 1147 (2001).

[54] J. Hoshen and R. Kopelman, Phys. Rev. B 14, 3438 (1976).

[55] To obtain the data of Fig. 4 consumed some 40 days of CPU time on a $3 \mathrm{GHz}$ AMD processor.

[56] N. B. Wilding, Phys. Rev. E 52, 602 (1995).

[57] We note that the two-peaked structure of the energy distribution persists to slightly larger values of $\epsilon^{-1}(\approx 0.43)$. However, the essential problem of identifying criticality remains.

[58] J. R. Errington, Phys. Rev. E 67, 012102 (2003).

[59] Y. Rosenfeld, Phys. Rev. Lett. 63, 980 (1989).

[60] Y. Rosenfeld, D. Levesque, and J.-J. Weis, J. Chem. Phys. 92, 6818 (1990).

[61] Y. Rosenfeld, J. Chem. Phys. 93, 4305 (1990).

[62] C. Caccamo, Phys. Rep. 274, 1 (1996).

[63] J. S. Høye, E. Lomba, and G. Stell, Mol. Phys. 79, 523 (1993).

[64] L. Belloni, J. Chem. Phys. 98, 8080 (1993).

[65] A. G. Schlijper, M. M. Telo da Gama, and P. G. Ferreira, J. Chem. Phys. 98, 1534 (1992).

[66] P. G. Ferreira, R. L. Carvalho, M. M. Telo da Gama, and A. G. Schlijper, J. Chem. Phys. 101, 594 (1994).

[67] M. C. Barbosa, Phys. Rev. E 48, 1744 (1993).

[68] L. G. MacDowell, V. K. Shen, and J. R. Errington, J. Chem. Phys. 125, 034705 (2006).

[69] The computational problems that stem from the large typical cluster size at criticality might be ameliorated by choosing a different amplitude of the repulsion such as to reduce the characteristic length scale of the cluster instability.

[70] F. J. Rogers and D. A. Young, Phys. Rev. A 30, 999 (1984). 